\title{
Valor nutricional do caldo de cana e potencial nutracêutico do caná - bebida
}

\section{fermentada do caldo de cana}

\author{
Nutritional value of cane broth and nutraceutical potential of caná - cane broth's fermented drink \\ Valor nutricional del caldo de caña y potencial nutraceutico del caná - bebida fermentada del caldo \\ de cana
}

Recebido: 24/01/2022 | Revisado: 15/02/2022 | Aceito: 16/02/2022 | Publicado: 25/02/2022

\author{
Amazile Biagioni Maia \\ ORCID: https://orcid.org/0000-0002-1119-8759 \\ Laboratório LABM, Brasil \\ E-mail: amazilebram@gmail.com
}

\begin{abstract}
Resumo
O Brasil é o maior produtor mundial de cana-de-açúcar, sendo o caldo destinado principalmente à produção de açúcar, álcool combustível e cachaça industrial. Em menor proporção, milhares de produtores cultivam cana de açúcar em escala rural, para produção da cachaça de alambique, açúcar mascavo, rapadura e melado. Nos últimos anos, esses produtores têm buscado opções que, além de ampliar o leque de produtos, permitam agregar mais valor às suas propriedades e canaviais. Nesse contexto, tem sido considerada a viabilidade de produção do caldo de cana fermentado, como bebida final, similar ao vinho de uvas. O potencial de mercado dessa bebida foi acentuadamente ampliado a partir de pesquisas recentes, que comprovaram a presença de fitoativos no caldo de cana, como a apigenina, a luteolina e a asterina, entre outros. Dar continuidade a esses trabalhos que já permitiram embasar cientificamente práticas milenares afetas ao emprego do caldo de cana na medicina ayuvédica, é muito importante, tanto para reforçar a valorização do caldo de cana na dieta humana, como para viabilizar a classificação do caná - o fermentado do caldo de cana emergente no Brasil como bebida com atividade funcional, a exemplo do reconhecimento já conquistado pelos vinhos que advêm da fermentação do suco das uvas. Para contribuir nesse sentido, apresenta-se uma atualização no âmbito da composição química do caldo de cana, destacando os principais fitoativos já caracterizados.
\end{abstract}

Palavras-chave: Cana de açúcar; Fitoquímicos; Caná; Caldo de cana fermentado.

\begin{abstract}
Brazil is the world's largest producer of sugarcane, and its juice is used mainly for the production of sugar, fuel alcohol and industrial cachaça. To a lesser extent, thousands of producers cultivate sugarcane on a rural scale, for the production of alembic cachaça, brown sugar, rapadura and molasses. In recent years, however, these producers have been looking for options that, in addition to expanding the range of products, allow them to add more value to their properties and sugarcane fields. In this context, the feasibility of producing fermented sugarcane juice as a final drink, similar to grape wine, has been considered. The market potential of this beverage has been markedly expanded as a result of recent research, which proved the presence of phytoactive substances in sugarcane juice, such as apigenin, luteolin and asterine, among others. Continuing these works, which have already made it possible to scientifically support millenary practices related to the use of sugarcane juice in Ayuvedic medicine, is very important, both to reinforce the appreciation of sugarcane juice in the human diet, and to enable the classification of sugarcane - the fermented sugarcane of the emerging sugarcane juice in Brazil - as a beverage with functional activity, following the example of the recognition already won by wines that come from the fermentation of grape juice. To contribute in this direction, an update is presented in the scope of the chemical composition of the sugarcane juice, highlighting the main phytoactives already characterized.
\end{abstract}

Keywords: Sugarcane; Phytochemicals; Caná; Fermented sugarcane juice.

\section{Resumen}

Brasil es el mayor productor mundial de caña de azúcar, y el jugo se utiliza principalmente para la producción de azúcar, alcohol combustible y cachaza industrial. En menor medida, miles de productores cultivan caña de azúcar a escala rural, para la producción de cachaza de alambique, panela, rapadura y melaza. En los últimos años, sin embargo, estos productores han estado buscando opciones que, además de ampliar la gama de productos, les permitan agregar más valor a sus propiedades y campos de caña de azúcar. En este contexto, se ha considerado la factibilidad de producir jugo de caña de azúcar fermentado como bebida final, similar al vino de uva. El potencial de mercado de esta bebida se ha ampliado notablemente como resultado de investigaciones recientes, que comprobaron la presencia de sustancias fitoactivas en el jugo de caña de azúcar, como apigenina, luteolina y asterina, entre otras. La continuación de estos trabajos, que ya han permitido sustentar científicamente prácticas milenarias relacionadas con el uso del jugo de caña de azúcar en la medicina ayuvédica, es muy importante, tanto para reforzar la apreciación del jugo de caña de azúcar en la 
dieta humana, como para posibilitar la clasificación de la caña de azúcar -la caña fermentada del jugo de caña emergente en Brasil- como una bebida con actividad funcional, siguiendo el ejemplo del reconocimiento ya ganado por los vinos que provienen de la fermentación del jugo de uva. Para contribuir en esa dirección, se presenta una actualización en el ámbito de la composición química del jugo de caña de azúcar, destacando los principales fitoactivos ya caracterizados.

Palabras clave: Caña de azúcar; Fitoquímicos; Caná; Jugo de caña fermentado.

\section{Introdução}

O caldo extraído pela moagem dos colmos da cana-de-açúcar (Saccharum spp) é saboroso e tem alto teor de glicídios. No Brasil, maior produtor mundial de cana-de-açúcar, o caldo é destinado principalmente à obtenção de açúcar e etanol combustível, além da cachaça - bebida destilada típica do Brasil (MAPA, 2021). O açúcar e o etanol combustível são fabricados predominantemente em grandes usinas e destilarias. Já a cachaça, além de destilada por algumas fábricas de grande porte (cachaça de coluna), é também produzida em escala agrícola por milhares de unidades disseminadas por todo o país (cachaça de alambique).

Com o avanço tecnológico das últimas décadas, muitos produtores da cachaça de alambique perceberam que o caldo de cana fermentado pode ter excelente palatabilidade, com potencial de inserção e grande aceitação no mercado. De fato, já foi demonstrada a viabilidade de aproveitamento da infraestrutura das fábricas de cachaça de alambique para a produção segura do fermentado de cana, como bebida estável e saudável (Maia, 2011; Almeida et al., 2021). Por analogia com o vinho, cujo nome remete à vinha, a bebida tem sido denominada "caná", que remete ao canavial (caná-vial).

A produção do caná tem potencial valorizar áreas geográficas (como o terroir dos vinhos), ampliar a viabilidade econômica e fortalecer o setor produtivo da cachaça de alambique, empregando recursos já disponíveis: matéria-prima, equipamentos, práticas e conhecimentos. Não obstante, é necessário que se perceba o caldo de cana de modo semelhante ao suco da uva, cujos teores de resveratrol e quercetina já se encontram popularizados como fatores de valorização nutricional dos vinhos (Sultana et al., 2008; Abdelaziz et al., 2015; Salehi et al., 2020).

Para isso, é necessário que se avance na composição minoritária do caldo de cana, evidenciando compostos que agreguem valores nutracêuticos. O objetivo desse artigo é apontar o estado da arte e estimular o desenvolvimento de mais pesquisas no âmbito dos componentes fitoativos do caldo de cana.

\section{Metodologia}

Foi feito o levantamento (Google Acadêmico, Scielo e Science.gov) das publicações referentes ao valor nutricional e fitoquímicos do caldo de cana, no período de 1990 até o presente momento. Os trabalhos incluíram artigos técnicos publicados em periódicos nacionais e internacionais, assim como monografias de mestrado e teses de doutorado em universidades brasileiras. A revisão foi escrita de modo conciso, com ênfase nos compostos fitoquímicos já identificados e que demandam maior aprofundamento científico no âmbito das variedades brasileiras de cana.

\section{Composição Centesimal}

O caldo de cana compõe-se majoritariamente de carboidratos, dentre os quais a sacarose representa em torno de $90 \%$. Em proporções bem menores, que variam na extensão do colmo, ocorrem também a frutose, glicose e ainda outros glicídios, como a rafinose (Pereira et al., 2017).

Dentre as variedades cultivadas no Brasil, a média do teor de carboidratos é 18,2 g/100 g caldo (TACO, 2011), acima da média de 13,2 g/100 g informada pelo IFCD (2017) para as variedades indianas. E sabe-se que, no ponto de maturação, as canas brasileiras atingem valores de até $24^{\circ}$ brix, que correspondem aproximadamente ao teor de carboidratos totais (em g/ 100 
g). Quantitativamente, os teores são similares aos das uvas; a diferença é estrutural, pois a frutose e a glicose é que são os açúcares predominantes nas uvas (Amerine et al., 1958; Orak,2009).

Os teores das demais classes que integram a composição centesimal variam de traços a 0,5 g/100 g (Tabela 1).

Tabela 1 - Composição centesimal do caldo de cana $(\mathrm{g} / 100 \mathrm{~g})$.

\begin{tabular}{ccc}
\hline COMPONENTE & BRASIL & ÍNDIA \\
$($ TACO, 2011) & $($ IFCD, 2017) \\
\hline Umidade & 81,7 & 86,0 \\
Carboidratos & 18,2 & 13,2 \\
Proteinas & Tr. & 0,2 \\
Cinzas & 0,1 & 0,2 \\
Gorduras totais & Tr. & 0,4 \\
Fibras totais & 0,1 & 0,5 \\
\hline
\end{tabular}

Fonte: Autora.

Quanto aos teores de vitaminas e minerais, dados da literatura permitem inferir que a ingestão diária de 200 $\mathrm{mL}$ de caldo corresponda a cerca de 10\% da IDR de piridoxina, ácido fólico e de ferro, assim como 30\% da IDR de vitamina $\mathrm{C}$, ácido pantotênico, riboflavina e tiamina. Os teores são relevantes, mas não chegam a caracterizar excepcionalidades frente a outros vegetais da nossa dieta habitual (TACO, 2011; Anvisa, 2005).

Face ao exposto, entende-se que, ao longo do século XX, a medicina ocidental tenha reconhecido o valor nutricional do caldo de cana apenas pela associação entre o alto conteúdo energético e potencial glicêmico atenuado devido ao predomínio do dissacarídeo sacarose (Iqbal et al., 2020). Embora recorrentes, as alegações de efeitos fitoterápicos eram atribuídas a "crendices populares" não fundamentadas.

\section{Propriedades Terapêuticas}

Ao longo de muitos séculos e até a presente dada, a medicina oriental (ayuvédica) sempre empregou o caldo de cana como agente de saúde e ação terapêutica no combate a diversas patologias. Mais recentemente, essas práticas têm se estendido a diversas aplicações veterinárias, com embasamento científico de pesquisadores asiáticos (especialmente da Índia, Turquia e China) e de outros países. Em frangos, por exemplo, foi demonstrada a eficácia da administração de doses medicinais (da ordem de $500 \mathrm{mg} / \mathrm{kg}$ peso/dia) para fins de:

- Efeito imunoestimulante e combate a infecções bacterianas (Abasy et al., 2002; Abasy et al., 2003; Akhtar et al., 2008);

- Ação protetora contra efeitos deletérios da radiação UV aplicada a frangos em cativeiro (Amer et al., 2005)

- Efeito protetor contra parasitas intestinais (Awaiss et al., 2011)

Com doses similares, testes na criação de porcos permitiram evidenciar:

- Efeito imunoestimulante e ação preventiva contra o vírus da pseudo-raiva, enfermidade infecto contagiosa grave (Lo et al., 2005, 2006)

- Efeito antioxidante mensurável na qualidade da carne de porco (Xia et al., 2017). 
Com testes em ratos, foi comprovado o efeito protetor contra a toxicidade renal aguda do antibiótico gentamicina, com indícios considerados promissores para o tratamento de infeções dos ossos, endocardite, doença inflamatória pélvica, meningite, pneumonia, infeções do trato urinário e sepse (Hussein et al., 2021).

\section{Fenólicos}

Os fenólicos - compostos que se caracterizam por conter um ou mais anéis aromáticos com um ou mais grupos hidroxila - são os metabólitos secundários mais abundantes das plantas vegetais. As estruturas de mais de 8.000 fenólicos começaram a ser desvendadas a partir de avanços relativamente recentes nos equipamentos e metodologias analíticas. $\mathrm{Na}$ sequência, inúmeras funções fisiológicas foram desvendadas, assim como os mecanismos moleculares envolvidos (Lee et al., 1998; Yoshikava et al., 2000; Machado et al., 2008 ; Abbas et al., 2014; Singh et al., 2015; Pimentel et al., 2019). Em especial, ácidos fenólicos e flavonoides (Figura 1) têm recebido grande atenção (Kumar et al.,2013; Kiokias et al., 2020).

Figura 1 - Esqueletos moleculares que caracterizam:

(a) ácidos fenólicos (C6-C1); (b) flavonoides (C6-C3-C6)<smiles>O=C(O)c1ccccc1O</smiles>

(a)<smiles>c1ccc(C2CCc3ccccc3O2)cc1</smiles>

(b)

Fonte: Autora.

No caldo da cana, já foi comprovada a presença de ácidos fenólicos e flavonoides importantes (Takara, 2002; Almeida et al., 2006; Kadam et al., 2008; Vila et al., 2008; Colombo et al., 2009), com destaque para os ácidos caféico, sinápico e clorogênico (Figura 2) e os flavonoides apigenina, luteolina, diosmetina, vitexina e tricina (Figura 3) .

Figura 2 - Ácidos fenólicos identificados no caldo de cana.

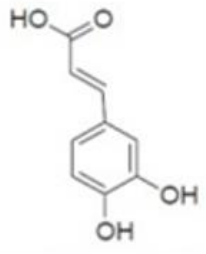

ácido cafeico

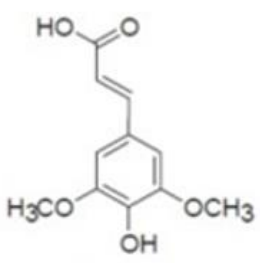

ácido sinápico

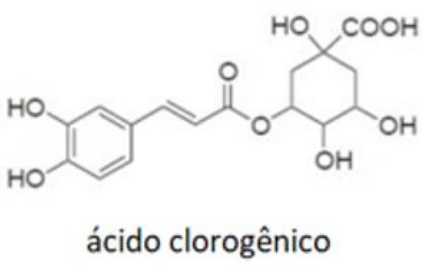

ácido clorogênico

Fonte: Autora. 
Figura 3 - Flavonoides já identificados no caldo de cana.

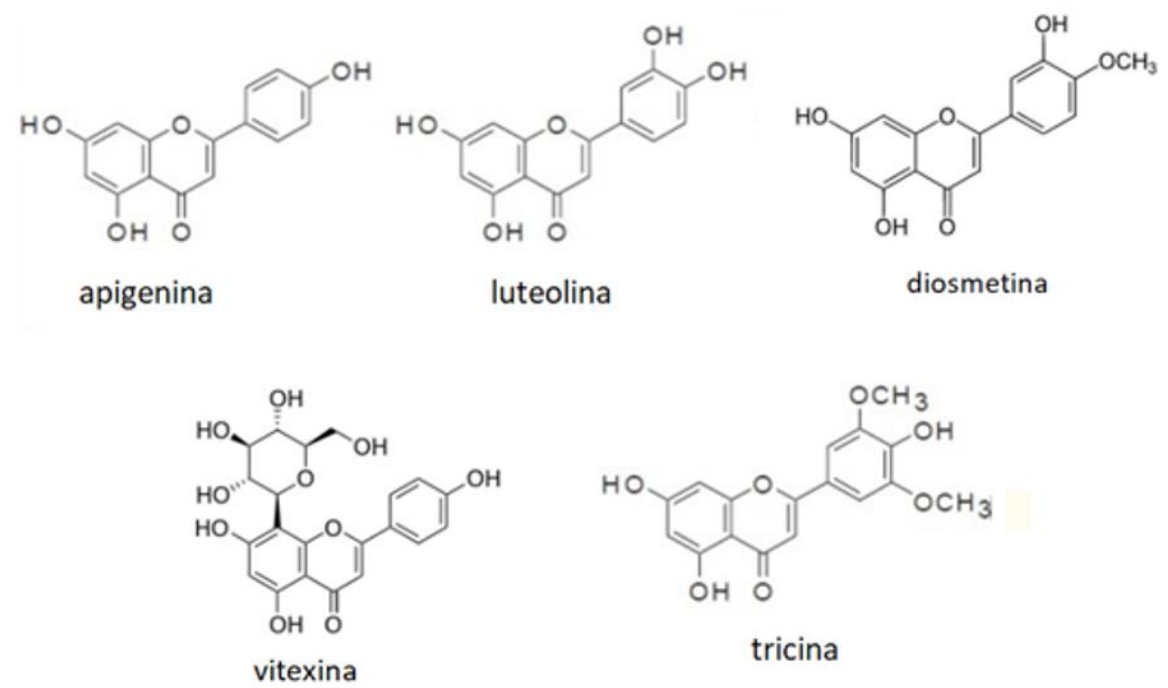

Fonte: Autora.

Os ácidos cafeico, sinapínico e clorogênico são amplamente reconhecidos por suas atividades antioxidantes e anticarcinogênicas, entre inúmeras outras funções biológicas no organismo humano e dos animais (Gulçin, 2006; Galano et al., 2011; Kim, 2019; Espíndola et al., 2019; Chen et al., 2016).

A apigenina e a luteolina são apontadas entre os antioxidantes mais potentes na classe dos flavonoides (Lee et al.,1998; Nielsen et al., 1999). Sua atividade antitumoral também foi comprovada (Jeyabal et al., 2005). Entre outros benefícios, Almeida et al. (2006) apontaram o potencial dos fenólicos da cana no combate o envenenamento por metilmercúrio (a forma mais tóxica do mercúrio).

\section{Antocianinas e Carotenoides}

Os flavonoides têm cor amarela (flavus, do latim, amarelo), à exceção das antocianinas, cuja tonalidade azul (do grego, anthos, flor e kianos, azul) pode contribuir para a cor verde do caldo fresco (Xu et al., 2022). Em estudo recente, Xu et al. (2022) apontaram a cianidina-3-O-glicosídeo (também chamada asterina) como principal representante das antocianinas no caldo de cana. Segundo Aguirre et al. (2016) a asterina tem evidências sólidas de eficácia antioxidante na proteção do DNA, ação gastro protetora, anti-inflamatória e antitrombótica; é também fator epigenético, exercendo proteção contra infecção por Helicobacter pylori, doenças relacionadas à idade, diabetes tipo 2, doenças cardiovasculares doença, síndrome metabólica e câncer bucal.

Figura 4 - Asterina, antocianina do caldo de cana.<smiles>O=C(c1ccc(O)c(O)c1)c1cc2c(O)cc(O)cc2[o+]c1OC1C(O)C(O)C2OC(CO)C(O)C1O2</smiles>

Fonte: Autora. 
O caldo de cana contém também carotenoides, que ocorrem nos cloroplastos, em associação com a clorofila (Schiozer \& Barata, 2009). Os carotenoides atuam com inúmeras funções no organismo humano, e muitos são precursores da vitamina A (Eggersdorfer et al., 2018). Ainda faltam estudos que esclareçam as estruturas peculiares em que ocorrem no caldo de cana. Até o momento, é relevante destacar que o caldo de cana tem sido amplamente pesquisado como meio para a fermentação por cepas geneticamente modificadas de Saccharomyces cerevisiae para fins de obtenção de alto rendimento em B-caroteno e outros carotenoides ( $\mathrm{Li}$ et al., 2017). Assim sendo, pode-se admitir que o Caná, como caldo de cana fermentado, tenha potencial para agregação de tecnologia associada a teores relevantes de carotenóides.

\section{Clorofila}

A cor verde do caldo de cana é atribuída principalmente ao seu teor de clorofila, cujos efeitos nutracêuticos são amplamente conhecidos (Hentschell, 2009; Ulbricht et al., 2014). O colmo da cana é rico em clorofila; no entanto, sua estrutura original é afetada em decorrência da moagem efetuada para extração do caldo, devido à desintegração dos cloroplastos (Schiozer et al., 2009). Além disso, sendo insolúvel em água, a clorofila tende a separar-se do caldo, por decantação natural ou em decorrência de procedimentos de clarificação. Esses fatores reduzem o potencial de relevância da clorofila original do caldo de cana relativamente a outras fontes vegetais.

\section{Considerações Finais}

O potencial nutracêutico do caldo de cana já foi apontado, com base na presença dos ácidos fenólicos cafeico, sinápico e clorogênico e dos flavonoides apigenina, luteolina, diosmetina, vitexina, tricina e asterina. Integrando a classe dos fitofenólicos, esses compostos têm despertado grande interesse científico, em todo o mundo - tanto para fins nutricionais como medicinais. No Brasil, as pesquisas têm sido esporádicas e aleatórias, sem evidenciar um empenho na valorização das dezenas de variedades cultivadas em toda a extensão do território brasileiro. No entanto, esses estudos são imprescindíveis para apoiar e validar a produção do caldo de cana fermentado (caná) conferindo-lhe status de valorização e reconhecimento semelhante ao do vinho. Por fim cabe destacar que, embora já exista em alguns países (Congo, Filipinas, Jamaica), a produção extensiva do caná a partir da fermentação do caldo de cana em centenas de alambiques de todo o país se revestirá de múltiplas peculiaridades que a caracterizarão, de fato, como a primeira bebida fermentada de concepção genuinamente brasileira.

\section{Referências}

Abbas, S.R., Sabir, S.M., Ahmad, S.D., Boligon, A.A. \& Athayde, M.L. (2014). Phenolic profile, antioxidant potential and DNA damage protecting activity of sugarcane (Saccharum officinarum). Food Chem. 147, 10-16.

Abasy, M., Motobu, M., Na, K.J, Sameshina, T., Koge, K. \& Onodera, T. (2002). Immunostimulating and growth promoting effects of sugarcane extracts (SCE) in chickens. J. Vet. Med. Science, 64, 1061-1063.

Abasy, M., Motobu, M., Na, K.J., Shimura, K., Nakamura, K. \& Koge, K. (2003). Protective effect of sugarcane ectracts (SCE) on Eimeria tenella infections in chickens. J. Vet. Med. Sci., 65, 865-871.

Abdelaziz, K.M., Cabrera, M.C.G., Vina, J. \& Borras, E.C. (2015). Properties of resveratrol: in vitro and in vivo studies about metabolism, bioavailability, and biological effects in animal models and humans. Oxid Med Cell Longev.: 837042. http://dx.doi.org/10.1155/2015/837042

Aguirre, F.O., García, J.R., Ruiz, N.M., Robles, A.C., Díaz, S.M., Parrilla, E.A. \& Medrano, A.W. (2016). Cyanidin-3-O-glucoside: physical-chemistry, foodomics and health effects. Molecules, 21(9). http://dx.doi.org/10.3390/molecules21091264

Akhtar, M., Hafeez, M.A., Muhammad, F. \& Haq, A.U. (2008). Immunomodulatory and protective effects of sugar cane juice in chickens against Eimeria Infection. Turkish J. Vet. Animal Sci., 32(6), 463-467.

Almeida, J.M.D., Novoa, A.V., Linares, A.F., Lajolo, F.M. \& Genovese, M.I. (2006). Antioxidant activity of phenolics compounds from sugar cane (Saccharum officinarum L.) juice. Plant Foods Human Nut., 61: 187-192. http://dx.doi.org/ 10.1007/s11130-006-0032-6 
Amer, S., Na, K.J., Motobu, M. \& Abasy, M. (2005). Radioprotective effect of sugar cane extract in chickens. Phytot. Res., 19(6):496-500. doi:10.1002/ptr.1688

Amerine, M.A. \& Thoukis, G. (1958). The glucose-fructose ratio of California Grapes. Vitis, 1, 224-229. doi.org/10.5073/vitis.1958.1.224-229

Anvisa (2005). Res. RDC n 269, de 22/09/ 2005. Aprova o Regulamento técnico sobre a ingestão diária recomendada (IDR) de proteína, vitaminas e minerais".

Awais, M.M., Akhtar, M., Muhammad, F, Haq, A.U. \& Anwar, I. (2011). Immunotherapeutic effects of some sugar cane (Saccharum officinarum L.) extracts against coccidiosis in industrial broiler chickens. Exp. Parasit.,128 (2),104-110. doi:10.1016/j.exppara.2011.02.024

Chen, C. (2016). Sinapic acid and its derivatives as medicine in oxidative stress-induced diseases and aging. Oxid Med Cell Longev. doi: $10.1155 / 2016 / 3571614$

Colombo, R; Yariwake; J.H., Queiroz, E.F. \& Hostettmann, K.N. (2009). On-line identification of minor flavones from sugarcane juice by lc/uv/ms and postcolumn derivatization. J. Braz. Chem. Soc., 20 (9). doi.org/10.1590/S0103-50532009000900003

Eggersdorfer, M. \& Wyss, A. (2018). Carotenoids in human nutrition and health. Arch. Biochem. Biophys., 652: 18-26. doi:10.1016/j.abb.2018.06.001

Espíndola, K.M.M., Ferreira, R.G., Narvaez, L.M.N., Rosario, A.C.R.S., Silva, A.H.M., Silva, A.G.B, Vieira, A.P.O \& Monteiro, M.C. (2019) Chemical and pharmacological aspects of caffeic acid and its activity in hepatocarcinoma. Front Oncol., 9, 541. doi: 10.3389/fonc.2019.00541

Galano, A.; Márquez, M.F. \& Idaboy, J.R.A. (2011). Mechanism and kinetics studies on the antioxidant activity of sinapinic acid. Phys. Chem. Chem. Phys., 13, 11199-11205. https://pubs.rsc.org/en/content/articlelanding/2011/cp/c1cp20722a

Gülçin, I. (2006). Antioxidant activity of caffeic acid (3,4-dihydroxycinnamic acid). Toxicol., 217(2-3):213-20. doi: 10.1016/j.tox.2005.09.011.

Hentschel, H. (2009). Considerações sobre a produção e utilização do caldo de cana. Agropec. Catarinense, 22 (2), 45-48. https://publicacoes.epagri.sc.gov.br/RAC/article/view/816

Hussein, Y.A. \& Shafey, R.S. (2019). The possible protective effects of saccharum officinarum 1. (sugar cane) juice co-supplementation on gentamicin induced acute renal toxicity in adult albino rats. Int. J. Pharm. Toxicology, 7 (2), 29-34. doi.org/10.14419/ijpt.v7i2.29477.

IFCD (2017) Indian Food Composition Tables. Composition of sugarcane juice (Saccharum officinarum). In: foodhttps://vikaspedia.in/health/nutrition/nutritive-value-of-foods/healing-effects-of-sugarcane-juice. doi-url: ifct2017.com/frame.php?page=home

Iqbal, A., Kamran, H., Khalid, S., Jabeen, S. \& Aslam, M. (2020). Glycemic response of natural sweeteners like sugarcane juice, honey and jaggery in healthy individuals. EAS J. Human. Cult. Studies, 2 (5). doi:10.36349/easjhcs.2020.v02i05.006

Jeyabal, P.V., Syed, M.B., Venkataraman, M., Sambandham, J.K. \& Sakthisekaran, D. (2005) Apigenin inhibits oxidative stress-induced macromolecular damage in N-nitrosodiethylamine (NDEA)induced hepatocellular carcinogenesis in Wistar albino rats. Mol. Carcinog. 44 (1): 11-20.

Kadam, U.S., Ghosh, S.B., Strayo, D. \& Suprasanna, P. (2008). Antioxidant activity in sugarcane juice and its protective role against radiation induced DNA damage. Food Chem., 106, 1154-1160.

Kim, J.K. \& Park, S.U. (2019). Chlorogenic acid and its role in biological functions: an up to date. Excli J.,18, 310-316. doi: 10.3390/molecules22030358

Kiokias, S., Proestos, C. \& Oreopoulou, V. (2020). Phenolic acids of plant origin - a review on their antioxidant activity in vitro (o/w emulsion systems) along with their in vivo health biochemical properties. Foods, 9(4) doi:10.3390/foods9040534

Kumar, S. \& Pandey, A.K. (2013). Chemistry and biological activities of flavonoids: an overview. Sci. World J., 1, 16. doi:10.1155/2013/162750

LABM (2021 ). Curso de tecnologia do caná -caldo de cana fermentado. Belo Horizonte, LABM. www.labm.com.br

Lee, S.K., Mbwambo, Z.H., Chung, H., Luyengi, L., Gamez, E.J., Mehta, R.G.; Kinghorn, A.D. \& Pezzuto, J.M. (1998). Evaluation of the antioxidant potential of natural products. Comb. Chem. High Throughput Screen , 1(1): 35-46.

Li, J., Shen, J., Sun, Z., Li, J., Li, C., Li, X., \& Zhang, Y. (2017). Discovery of several novel targets that enhance $\beta$-carotene production in Saccharomyces cerevisiae. Frontiers in Microbiology, 8. doi:10.3389/fmicb.2017.01116

Lo, D.Y., Chen, T.H., Chien, M.S., Koge, K., Hosono, A. \& Kaminogawa, S. (2005). Effects of sugarcane extract on modulation of immunity in pigs. J. Vet. Med. Science, 67(6), 591-597.

Lo, D.Y., Chien, M.S., Yeh, K.S., Koge, K., Lin, C.C., Hsuan, S.L. \& Lee, W.C. (2006). Effects of sugar cane extract on pseudorabies virus challenge of pigs. J. Vet. Med. Sci., 68(3): 219-225. doi: 10.1292/JVMS.68.219.

Machado, H., Nagem, T.J., Peters, V.M., Fonseca, C.S. \& Oliveira, T.T. (2008). Flavonóides e seu potencial terapêutico. Bol. Centro Bio 1. Reprod. (Juiz de Fora), $27(1 / 2), 33-39$.

MAPA (2021). Portaria 339/21 de 28/06/2021. Estabelece os Padrões de Identidade e Qualidade da aguardente de cana e da Cachaça e revoga atos normativos com matérias pertinentes.

Orak, H.H. (2009). Glucose and fructose contents of some important red grape varieties by HPLC. Asian J. Chem, 21(4): 3068-3072.

Pereira, L.F.M., Ferreira, V.M., Oliveira, N.G., Sarmento, P.I.V.S.; Teodoro, E. (2017). Sugars levels of four sugarcane genotypes in different stem portions during the maturation phase. An. Acad. Bras. Cienc., 89 (2). doi.org/10.1590/0001-3765201720160594 
Research, Society and Development, v. 11, n. 3, e33811326112, 2022

(CC BY 4.0) | ISSN 2525-3409 | DOI: http://dx.doi.org/10.33448/rsd-v11i3.26112

Pimentel, C.V.M.B., Elias, M.F. \& Philippi, S.T. (2019). Alimentos funcionais e compostos bioativos. São Paulo, Manole. 963 p. ISBN-13: 978-8520453605

Salehi, B., Machin, L., Monzote, L., Rad, J.S., Ezzat, S.M., Salem, M.A., Merghany, R.M., Mahdy, N.M., Kiliç, C.S., Sytar, O., Rad, M.M., Sharopov, F., Martins, N, Martorel, L.M. \& Cho, W.C. (2020). Therapeutic potential of quercetin: new insights and perspectives for human health. ACS Omega, 5 (20): 11849-11872. https://doi.org/10.1021/acsomega.0c01818

Schiozer, A.L. \& Barata, L.E.S.; (2007). Estabilidade de corantes e pigmentos de origem vegetal. Rev. Fitos, 3(2), 6-24.

Singh, A., Lal, U.R.; Mukhtar, H.M.; Singh, P.S.; Shah, G. \& Dhawan, R.K. (2015). Perfil fitoquímico da cana-de-açúcar e seus potenciais aspectos à saúde. Pharmacogn Rev., 9 (17), 45-54. doi: 10.4103 / 0973-7847.156340

Sultana, B. \& Anwar, F. (2008) Flavonols (kaempeferol, quercetin, myricetin) contents of selected fruits, vegetables and medicinal plants. Food Chem, 108, 879-888.

TACO - Tabela Brasileira de Composição de Alimentos (2011). Campinas, cfn.org.br/wp-content/uploads/2017/03/ taco_4_edicao_.pdf

Takara, K., Matsui, D., Wada, K., Ichiba, T. \& Nakasone, Y. (2002). New antioxidative phenolic glycosides isolated from Kokuto noncentrifuged cane sugar. Biosci. Biotec. Biochem., 66, 29-35.

Ulbricht, C., Bramwell, R., Catapang, M., Giese, N., Isaac, R., Le, T.D. \& Zeolla, M.M. (2014). An evidence-based systematic review of chlorophyll by the natural standard research collaboration. J. Diet. Supplements, 11(2), 198-239. doi:10.3109/19390211.2013.859853

Vila, F.C.; Colombo, R.; Lira, T.O. \& Yariwake, J.H. (2008). HPLC microfractionation of flavones and antioxidant (radical scavenging) activity of Saccharum officinarum L. J. Braz. Chem. Soc., 19(5), 903-908.

Xia, Y., Li, Y., Shen, X., Mizu, M., Furuta, T. \& LI, C. (2017). Effect of dietary supplementation with sugar cane extract on meat quality and oxidative stability in finishing pigs. Animal Nutrition, 3(3), 295-299. doi.org/10.1016/j.aninu.2017.05.002

Xu, Z., Wang, C., Yan, H., Zhao, Z., You, L. \& Ho, C.T. (2022). Influence of phenolic acids/aldehydes on color intensification of cyanidin-3-O-glucoside, the main anthocyanin in sugarcane (Saccharum officinarum L.). Food Chem, 30, 373. doi: 10.1016 / j.foodchem.2021.131396

Yamano, S., Ishii, T., Nakagawa, M., Ikenaga, H. \& Misawa, N. (1994). Metabolic engineering for production of beta-carotene and lycopene in Saccharomyces cerevisiae. Biosci Biotechnol Biochem, 58 (6), 1112-4. doi: 10.1271/bbb.58.1112.

Yoshikava, T., Toyokuni, S., Yamamoto, Y. \& Naito, Y. (2000). Free radicals in chemistry, biology and medicine. OICA International London. doi:10.1093/acprof:oso/9780198717478.001.0001 